\title{
ZUR ROMANISCHEN UND DEUTSCHEN RHYTHMIK.
}

Beitr. 23, 66 ff. habe ich versucht nachzuweisen, dass der begriff 'zehnsilbler' in der romanischen rhythmik mehrdeutig sei. Hinter einem vers der im text 10 silben aufweist, können die rhythmischen formen des sechsers und des vierers - dieser in gepresster art - stehen. Es handelt sich nun darum, den wichtigen rhythmischen unterschied schon aus dem text zu erkennen. Auf s. 79 ist gezeigt, wie dazu die sogenannte 'cäsur' - nach genauer terminologie vielmehr 'binnencäsur' zu nennen (vgl. a. a.o. s. $47, \S 8$. s. 49, §10) - und ihre behandlung dienen kann.

Der sechser - $-\dot{-} ;-\ddot{-}-\dot{-}-\dot{\sim}$ (bez. $\dot{\sim} \pi)$ ist eine weit verbreitete und sehr einfache form. Er hat als primäre reihe einen verhältnismässig schwachen einschnitt. Im text braucht derselbe nur leicht (durch wortschluss) angedentet zu sein; zuweilen wird er völlig übergangen, so dass nur die modulation der ganzen reihe die grundteilung erkennen lässt. Man spricht im letzten fall von 'versen ohne (binnen-)cäsur'; nicht ganz richtig, man müsste denn 'cäsur' geradezu als 'einschnitt' verstehen. Der vierer, das dekasyllabon (s. 75 ff.), hat als secundäre reihe eine viel stärkere binnencäsur: sie ist unter allen umständen ein einschnitt und darum im text stets zu sehen. Denn sie ist, wie s. 77 zeigt, aus einer früheren 'cäsur' entstanden dadurch, dass die periode von 2 gliedern, jedes zu 4 takten (bez. füssen) in ein viertaktiges glied, von 2 abschnitten umgewandelt ist. Die grenze zwischen den gliedern, d.h. die 'cäsur', wurde somit zu einer grenze zwischen abschnitten (vgl. s. 47, § 8), d. h. einer 'binnencäsur'. Diese umwandlung heisst 'pressung' und ist ein vorgang, aus dem 
allein sich die eigentümlichen formen erklären, die die moderne instrumentalmusik vorzugsweise benutzt und die der antiken rhythmik ganz fremd sind. Die hauptform des dekasyllabons

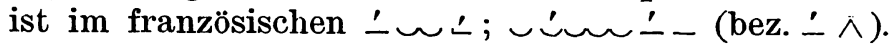

Der anschaulichkeit wegen mögen hier einige analysen dekasyllabischer strophen folgen. Durch ligaturen (im schema durch $\frown$ angedeutet) und einmischung anderer verstypen wird das bild gelegentlich ein wenig verdunkelt.

Béranger, Le coin de l'amitié, in Musique des chansons de Béranger, Paris, Garnier frères, s. 14:

L'amour, l'hymen, l'intérêt, la folie, aux quatre coins se disputent nos jours:

L'amitié vient compléter la partie; mais qu'on lui fait de mauvais tours!

Lors qu'aux plaisirs l'âme se livre entière, notre raison ne brille qu'à moitié,

Et la Folie attaque la première

le coin de l'amitié, le coin de l'amitié,

le coin de l'amitié.

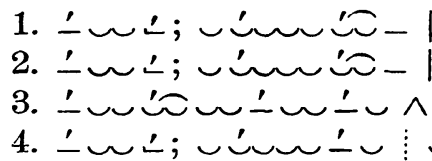

Rh. schema: $1 a-b$

$2 a-b$.

$3 a-b$

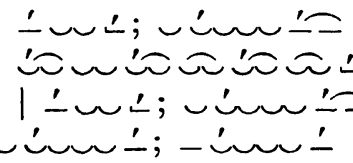

(achtsilbler!)

$4 a-b-b^{\prime}$

Reimschema: $a-b$

$a-b$.

$\mathrm{c}-\mathrm{d}$

$c-\delta+d-d$

Das dekasyllabon ist rein oder fast rein in zeile $1 \mathrm{a}, 1 \mathrm{~b}, 2 \mathrm{a}$, $3 \mathrm{~b}, 4 \mathrm{a}$ des rhythmischen schemas zu sehen. $3 \mathrm{a}$ ist der zehnsilbler auf einen andern gepressten rhythmus componiert: in $2 \mathrm{~b}$ ist ein achtsilbler mit vielen ligaturen so weit gestreckt, dass er für einen gepressten vierer ausreicht. Der primäre vierer würde in der umgebung von pressrhythmen stïren. Derartige. freiheiten sind erst neueren stiles: einfluss der instrumentalmusik liegt darin vor. - Die vierte periode ist dreigliedrig. Aus der melodie folgt, dass der letzte sechssillbler ein b'. d. h. eine schlusswiderholung ist. Vgl. R.hythm. \$ 10 (s. 19). Iuch diese zeile ist, hier anf einen gejressten rhythmus garogrin

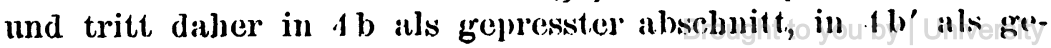


resste dipodische reihe auf. Die primäre form des sechsers

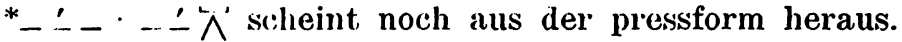

Andere modificationen des dekasyllabons zeigt ebda. s. 173: 'Treize à table:

Dieu, unes aunis, nous sommes treize à table, et devant moi le sel est répandu.

Nombre fatal! présage épouvantable!

la mort accourt: je frissonne éperdu, la mort accourt: je frissonne éperdu. Elle apparaît, esprit, fée ou déesse; mais, belle et jeune, elle sourit d'abord, mais, belle et jeune, elle sourit d'abord. De vos chansons ranimez l'allégresse; non, mes amis, je ne crains plus la mort, De vos chansons ranimez l'allégresse non mes amis, je ne crains plus la mort.

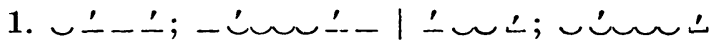

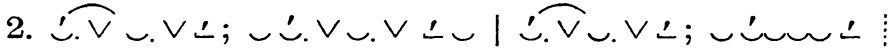

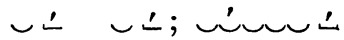

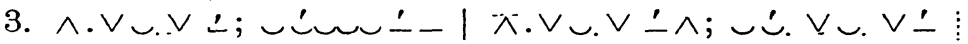

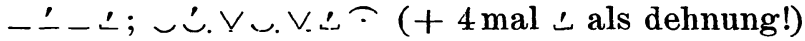

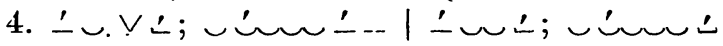

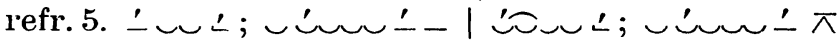

Rh. schema: $1 a-b$

$$
\begin{aligned}
& 2 a-b-b^{\prime} . \\
& 3 a-b-b^{\prime} \\
& 4 a-b
\end{aligned}
$$

refr. $5 a-b$
Reimschema: $a-b$

$$
\begin{aligned}
& a-b-b . \\
& c-d-d \\
& c-d
\end{aligned}
$$

refr. $c-d$

Zur erläuterung des rhythmischen schemas vgl. verf., Zur metrik Otfrieds v. Weissenburg (Festschrift für Sievers, 1896) s. 183. $\smile=$ kürze, $\vee=1 / \imath \smile=$ halbkürze, $\smile=1 \mathrm{kürze}+$ $1 / .2$ kürze; $\wedge=$ pause vom wert $\smile, \wedge^{*}=$ pause vom wert $\smile \cdot$. bezeichnet die cäsur, wenn die zusammenstossenden reihen des taktes wegen verkürzungen oder verlängerungen über ihren ursprünglichen wert erlitten haben. - In reihe $3 \mathbf{a} . \mathbf{b}$ habe ich der übersicht wegen die pausen an den anfang gestellt. Streng genommen muss man sie an das anhängen, was ihnen vorausgeht. Doch kommt darauf hier nichts an. Im notentext wird $\smile$ durch $\curvearrowright, \vee$ also durch $\curvearrowright, \smile \cdot$ durch $\curvearrowright$. vertreten. 
Interessant ist dies lied, weil hier die melodie öfters die früher s. 79 oben besprochene form des dekasyllabons zeigt,

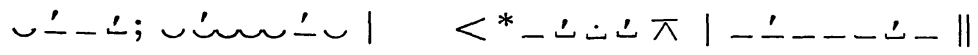

wenn auch ein wenig modificiert. So in reihe $1 \mathrm{a}, 2 \mathrm{~b}^{\prime}, 3 \mathrm{~b}^{\prime}$.

Die form $3 \mathrm{a}, 3 \mathrm{~b}$ ist in der heutigen musik sehr beliebt. Sie ist secundär entwickelt.

Die binnencäsur des dekasyllabons ist schon ihrer entstehung wegen rhythmisch unvergleichlich schärfer als die des einfachen sechsers, der eine primäre bildung ist. Man erwartet, dass sich auch im romanischen worttext der unterschied der rhythmischen grenzen bemerklich macht. Das ist, wie es scheint, wirklich der fall. Darauf möchte ich eben hier hinweisen.

Ich habe schon a. a. o. s. 79 die sog. epische cäsur des zehnsilblers herangezogen. Die scheinbar überschlagende silbe erklärt sich nur aus dem dekasyllabon (vgl. s. 78), zeugt also für dieses, wo sie sich findet. Im sechser würde sie zwingen anzunehmen, dass die dritte senkung aufgelöst sei und das ist unmöglich. Denn 'auflösung' oder genauer die besetzung zweier aufeinander folgender kürzen der melodie (in der function von masszeiten; $\sim$ ) mit je einer sprachsilbe kennt die mittelalterliche romanische verskunst nicht. Sie braucht nur die ligatur, d. h. zwei') kürzen der melodie auf eine silbe des textes.

A. a. o. s. 79 habe ich auch angenommen, es gäbe dekasyllaben mit überschlagender weiblicher silbe hinter der dritten. Ein vers wie et a Lengres seroic malbaillis wurde rhythmisiert:

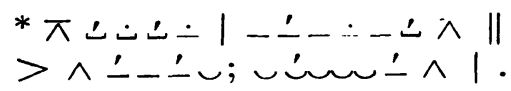

Nun würde sich ein contrapunktiker oder ein moderner nperncomponist gewis nicht scheuen verse mit jener sog. lyrischen binnencäsur so zu componieren. Denn das alte romanische rhythmische system ist durch die polyphonie und jetzt durch den einfluss des instrumentalen stiles sehr verändert worden. Aber für die ältere zeit, d. h. die zeit der herschaft des homo-

1) Auch mehr als zwei töne werden liirrt, namentlich wemn sir den charakter von verzierungen tragen. 
phomon voralen stiles solshe formen anzunehmen ist nicht zulïssien, Weil dies dem princip der silbenzählung widerspricht. Die silhe -!ress schicsst tatsüchlich nicht über, sondern zählt mit, sic: ist \%wal dem sprachaccent nach unbetont, aber dennoch rhythmisch eine 'tonsilbe'; vgl. 'l'o bler'2 s. 85. Stengel, Gröbers (irundr: :2, 1, 1, s. $51 \mathrm{f}$.

Num ist klar, dass eine binnencäsur sehr schwach sein muss, wenn man unmittelbar vor sie eine unbetonte sprachsilbe stellen darf. Denn starke rhythmische einschnitte, also diäresen, cäsuren, binnencäsuren von pressreihen haben immer eine auch sprachlich betonte tonsilbe vor sich. Ist nun in einem textzehnsilbler die binnencäsur sehr schwach, so liegt nahe, hinter ihm nicht das dekasyllabon, sondern den sechser zu suchen. Somit wäre die sog. lyrische binnencäsur des zehnsilbler's ein kriterium für den sechser, wie es die epische für das dekasyllabon ist.

Das scheint nach den angaben die von 'Tobler und Stengel gemacht werden, wirklich der fall zu sein. Das dekasyllabon ist der rhythmus der chansons de geste. Daher ist in der epik und überhaupt der erzählenden afrz. dichtung die epische binnencäsur beliebt (Stengel s.50). Diese wird dagegen und, wie Stengel sagt, von anfang an in der nord- und südfranzösischen lyrik gemieden. Nicht 'weil in folge des einheitlich gestalteten tonsatzes der versmelodie die pause im innern der einzelnen verse nicht mehr zur geltung kam, zehnsilbler also auch dem baue nach wie einreihige verse behandelt werden mussten' (Stengel s. 50). Denn der zehnsilbler ist in jeder form eine reihe wie der achtsilbler und andere verse. Sondern der lyrische zehnsilbler wird eben in weitaus den meisten fällen rhythmisch etwas anderes gewesen sein als der epische: dieser das dekasyllabon, jener der sechser.

Dass das ursprünglich gewis französische dekasyllabon zunächst in die französische, dann auch in die provenzalische lyrik eingedrungen sei, ist wahrscheinlich. Umgekehrt kann man den erzählervers gelegentlich nach dem schema des lyrischen gebaut haben, um so leichter, wenn es sich dabei um sprechpoesie handelte; vgl. Stengel $§ 108$.

Einen sicheren beweis für den sechser darf man in der 
binnencäsur sehen, die Stengel $\S 109$ 'die schwache' nennt. Ihr schema ist

$$
\text { -ヘーー-ー; }
$$

qui de s'amie respite sa joie qu'elle te face bien sovent chanteir

(Tobl. s. 86); vgl. auch meine abhandlung s. 79 f. Es handelt sich da lediglich um den sechser mit 'verschobener' binnencäsur (ebda. s. 52. Rhythm. § 20). Diese binnencäsur brauchen die Griechen z.b. im iambischen trimeter, der bekanntlich ein (freilich akatalektischer) sechser ist:

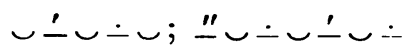

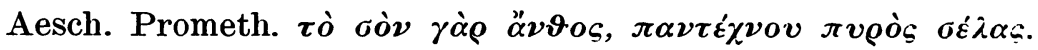

Es ist sehr bezeichnend, dass diese 'schwache' binnencäsur nicht in der epik, sondern nur in der lyrik vorkommt (Tobl. s. 86). Wenn sie auch selten ist, so beweist sie doch für den rhythmus der zehnsilbler, unter denen sie sich findet, sehr viel. Dass Stengel s. 53 solche verse nicht cäsurlos nennen will, ist durchaus berechtigt. Wenigstens haben die von Tobler s. 86 mitgeteilten verse alle einen wenn auch schwachen einschnitt. Man darf dessen stärke aber nicht an der binnencäsur des dekasyllabons messen.

Zehnsilbler ohne binnencäsur, die Tobler s. $87 \mathrm{f}$. mit recht annimmt, können nur sechser sein, wie ich schon betont habe (s. 80). Die binnencäsur einer pressreihe fällt, so weit ich sehe, nicht einmal in der sehr freien modernen instrumentalmusik weg. Dagegen haben sechser ohne einen im text ausgeprägten einschnitt rhythmisch nichts ungewöhnliches. Tobler rechnet mit recht dazu auch die fälle, wo in liedern (nicht im epos) der zehnsilbler hinter der sechsten betonten einen einschnitt hat. Man vergleiche hierzu fälle wie Aeschylos

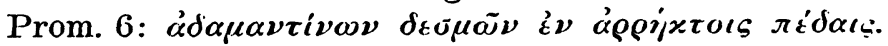

Im epos, auch den romanzen 1,5 und 1,16 (Bartsch) sind solche verse ganz anders zu beurteilen ('Tobler s. 87. Ntengel \$110). Dort gibt es einen typus, in dem sich die silhen in $6+4$ gruppieren. Dabei ist die sechste syrachlich und rhythmisch eine tonsilbe und dahinter liegt eine anch im text seharf ausgeprägte binnencïsur. Z. b. 17.0m. ma past. 1. 5 lon sumedi 
"soir, fint la srmminne. Hinter der betonten sechsten findet sich oft eine 'iiberschiessende' silbe, wie im dekasyllabon hinter der betonten vierten.

J. b. ebda. v. 12 reva toi an arriere, bien seis la vile. Eben dies beweist schlagend, dass wir es hier nicht mit einer pentapodie oder einem zweigliedrigen langvers zu tun haben, sonler'n, wie Stengel s. 53 richtig erkennt, mit einer versform, die wan als eine andere combination der teile (abschnitte) des gewölınlichen epischen zehnsilblers auffassen muss. In diesem gruppieren sich die silben wie $4+6$

z. b. Charles li reis nostre emperere magnes;

in jenem umgekehrt $6+4$

z. b. L'anfes Gerairs et Gaie s'an sont torneit.

Diese gebilde sind also pressrhythmen und abarten des dekasyllabons, d. h. gepresste vierer.

Ihr zweiter abschnitt ist im rhythmus

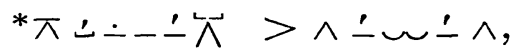

vielleicht auch

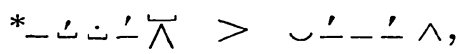

eine form die auch heute noch in liedern vorkommt.

Ein beispiel wird es veranschaulichen, nämlich die schlusszeile des bekannten liedes 'Hinaus in die ferne mit lautem hörnerklang', Lahrer commersbuch no. 52 (s. 57). Ich analysiere die erste strophe ganz.

refr. 3 . $\smile \div \div \div$
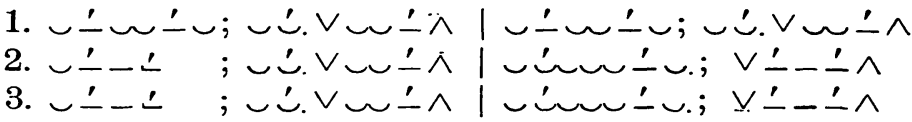

$$
\begin{array}{ll}
\text { Rhythm. schema } & 1 \mathrm{a}-\mathrm{b} \\
& 2 \mathrm{a}-\mathrm{b} \\
& 3 \mathrm{a}-\mathrm{b} .
\end{array}
$$

Der rhythmus ist zweifellos eine weiterbildung des französischen dekasyllabons. 2a, 3 a sind zehnsilbig und haben die früher s. 79 oben erläuterte form, nur mit gelegentlich punktierten werten. 1 a. b haben vom französischen standpunkt aus gesehen die 'epische binnencäsur'; nämlich die kürze hinter der zweiten thesis ( $(\dot{)})$ ist 'weiblich'. Ausserdem haben sie nach deutschem 
brauch 'auftakt', d.h. die erste arsis bleibt. Denkt man die reihenauftakte weg, dann hat man genau den rhythmus des epischen dekasyllabons mit 'epischer' binnencäsur.

$2 \mathrm{~b}$. $3 \mathrm{~b}$ sind nun dekasyllaben der gruppierung $6+4 \mathrm{mit}$ 'epischer' binnencäsur; der wert der 'überschiessenden' silbe tritt hier zufällig punktiert ( $\bullet$.) auf.

Im hinblick auf diese schlusszeile könnte man die romanze 1, 5 bei Bartsch sehr wol so analysieren:

Lou samedi a soir, fat la semainne

Gaiete et Oriour, serors germainnes, main et main vout bagnier a la fontainne.

Vante l'ore et li raim crollent:

ni s'antraimment soweif dorment.

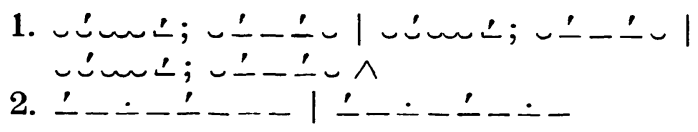

Schema $1 a-a^{\prime}-b$.

$2 a-b$.

Die form ist gewis alt, aber dass sie älter als der normale zehnsilbler $(4+6)$, ja ihm gegenüber ursprünglich sei, wie Stengel will (§ 110), halte ich für unwahrscheinlich. Jedenfalls reichen die beweise Stengels nicht aus, seine annahme zu sichern. Mit dem endecasillabo der Italiener hat der rhythmus nichts zu tun, denn der italienische vers ist ein sechser, was seine binnencäsur beweist.

Nach alledem scheint es, als ob wir für die älteste zeit $\mathrm{zu}$ scheiden hätten den epischen zehnsilbler $(4+6,6+4)$ und den lyrischen. Ersterer ist ein gepresster vierer (dekasyllabon), letzterer ein sechser primärer bildung. Erst allmählich dringt das dekasyllabon in die franzüsische und provenzalische lyrik ein und beeinflusst umgekehrt der sechser hier und da den epischen versbau. Entscheiden kann im einzelnen fall nur eine umfassende untersuchung.

Für die entscheidung dürfte ausser der beobachtung der formen der binnenciisur von wichtigkeit sein 70 ermitteln. wie die einschnitte im text syntaktisch hergestellt werden. I)'mn sowol das dekasyllabon wie der sechser haben im romanisele'n der regel nach hinter der vierten betonten silbe einen deullichen 
rinschnitt. Sie unterscheiden sich also fürs auge nicht gerade solir. Stengrel stellt s. 51. ff. darüber mancherlei zusammen. Von vorn herein ist zu erwarten, dass die binnencäsur des dokasyllabons durch syntaktisch stärkere einschnitte festgelegt wird, dass dagegen die binnencïsur des sechsers i. a. schwächer ist. lemmach müsste im allgemeinen das epos relativ starke, dic lyrik dagegen relativ schwache syntaktische einschnitte vorziehen. lenn sie liebt ja den sechser, wenn die obigen rrwägungen richtig sind.

Die beobachtungen die man bisher gemacht, stimmen dazu. Stengel $\$ 113$ teilt mit, dass schon in der altfranzösischen lyrik die binnencäsur rhythmisch und syntaktisch nachlässig behandelt, ja mehrfach geradezu verwischt werde. Noch schwächer als die gleichzeitigen altfranzösischen dichter markierten die Provenzalen die binnencäsur (Stengel $§ 116$ ). Bei den Italienern könne von syntaktischer ausprägung der binnencäsur überhaupt keine rede sein ( $(117)$. Alles das weist auf den sechser.

Ich füge noch einige nachträge und verbesserungen zu meiner früheren abhandlung hinzu.

S. 44 zeile 11 v. o. lies Aristoxenos' bd. 2.

$\mathrm{Zu}$ s. 48, § 9. Es ist ein hauptfehler in Westphals Analysen von minneliedern, dass er glaubt, eine reihe könne in ihnen isoliert stehen. So in dem liede Walthers, das er Allgemeine theorie s. 251 analysiert. Auch in seinen arbeiten über antike rhythmik ist dieser punkt vernachlässigt. Die theorie der periode leidet sogar in Rossbachs darstellung der antiken strophenformen (bd. 3 der Theorie der musischen künste der Hellenen) aus eben demselben grunde an unklarheit. Isolierte reihen sind in aller strophik, überhaupt den sog. geschlossenen formen nur in wenigen, als solche zudem leicht verständlichen fällen zuzulassen. Namentlich kommen instrumentale vor- und zwischenspiele, auch clauseln in betracht. Im griech. chorlied halte ich alleinstehende kola für ausgeschlossen, wenigstens im vocalen teil, von dem wir allein wissen.

Die anm. 2 auf s. 50 gehört zu $§ 16$.

$\mathrm{Zu}$ s. 69 unten kann ich jetzt auf den sehr wichtigen aufsatz von H. Ri e mann, Die melodik d. minnesinger, Fritzsches Musik. wochenbl. 28, s. $449 \mathrm{ff}$. verweisen (besprochen im.Jahresber. 
für german. philol. 19, 235 f.). Riemann hat dort nachgewiesen, dass die melodien der romanischen und deutschen minnesinger nicht mensural, sondern choral notiert sind. Die notenzeichen der hss. geben also keine zeitwerte (longae, breves u.s.w.), sondern bloss tonhöhen wie die griechischen notenbuchstaben. Es kann darum aus den melodien kaum etwas über den rhythmus der lieder entnommen werden. Der rhythmiker ist auf betrachtung des textes angewiesen nnd muss so vorgehen, wie es in den abschnitten III-v dieser untersuchung geschieht. Dennoch ist es gerade für romanische lieder wichtig, die melodien zu kennen. Denn wie Riemann gefunden (s. 450), bestätigt eben die melodieführung die vor Quicherat und seinen anhängern herschende ansicht der metriker, dass die alten romanischen verse alternierenden rhythmus hätten, eine ansicht der ich oben s. 69 aus allgemeinen gründen und auf grund mhd. nachahmungen romanischer formen beigetreten bin (s. 71 -73). Man wird nun wol aufhören, die einfachen und doch gefälligen rhythmen der alten lieder mensural zu verderben. Die rhythmisierung altfranzösischer und provenzalischer strophen, die oben versucht ist, hat so von ganz anderer seite her willkommene bestätigung empfangen.

Ebenso hat Riemann unabhängig von Eickhoff und mir (vgl. oben s. 75 ff.) die eigentümliche form entdeckt, die ich der kürze wegen dekasyllabon genannt habe, einen rhythmus der eben deshalb merkwürdig ist, weil er gegen den gebrauch der iibrigen romanischen verse nicht immer alterniert. Warum er das nicht tut, ist s. 77 erklärt. Ueber die mängel der Riemannschen arbeit vgl. Jahresber. s. 236.

Uebrigens sei hier angemerkt, dass dasselbe was Riemann für die minnelieder nachweist, erst recht für die homophonen melodien gilt, die der früheren zeit angehören. $\mathrm{Ks}$ ist nicht zulässig z. b. Ratperts lobgesang mit MSI)3 s. 84 in $3_{/ 4}$ takt zu bringen. Auch diese melodie ist choral notiert (neumiert) und die spätere mensurale zeitteilung, deren regeln .Tacolosthal ermittelt, auf sie anzuwenden, ist eben darum nicht stathaft. Neunen haben keine mensur. Tripeltakt ist später froilioh von den mensuralisten stark bevorzugt, ja als der cinzigr mörgliche angesehen worden. Fiir die ailtere zeit heweist das nichts. 
Der lateinische text ist vermutlich folgendermassen $\mathrm{zu}$ analysieren, wobei dis taktart unsicher bleibt'):

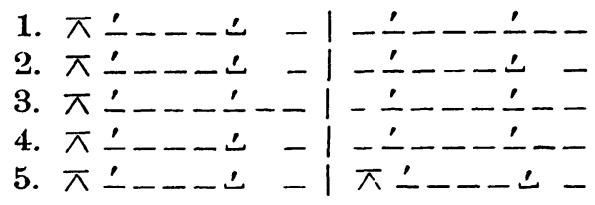

Alle verse schliessen thetisch, wie bis zum minnesang die ahd. mhd. reihen überhaupt. Die vorderglieder entbehren meist des auftakts.

Ebensowenig ist das Petruslied mensuriert; anders, wie es scheint, bei Scherer s. 63. Das schema ist wol ${ }^{\prime}$ ):

MSD. IX

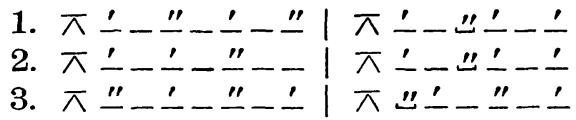

Die ictenabstufung nach Sievers' typen. Die glieder entbehren meist des auftakts.

Hierher gehören auch die sequenzenformen no.xrx-xxII, die gleichsam 'freie rhythmen' sind. Man vergleiche hierzu die darstellung der byzantinischen rhythmik bei Christ, Anthologia graeca. Dass die rhythmische prosa dieser sequenzen öfters versmässig, metrisch gesteigert wird, fällt nicht auf. Es ist das eine natürliche entwicklung der 'freien rhythmen', welche die nhd. poesie auch kennt. Der rhythmus dieser 'modi' ist natürlich der gregorianische, der sich an den sprachlichen anschliesst. Vgl. X. Haberl, Magister choralis $10^{5}$ (1893), s. 1 und s. $186 \mathrm{f}$. $197 \mathrm{ff}$. Ich werde anderswo genauer auf diese rhythmen zurückkommen.

Zu s. 78. Bei der ableitung der dekasyllaben wäre es klarer gewesen, statt der formen die aus dem wechselnden zusammenhang der compositionen genommen sind, zunächst

1) Da man es hier mit geistlichen werken, nicht solchen weltlichen ursprungs zu tun hat, so bleibt auch die rhythmusart unsicher. Denn diese geistliche vocalmusik kann immer von dem freien gregorianischen rhythmus beeinflusst sein. Dann hat die frage nach der taktart überhaupt keinen sinn. 
die historisch genau entsprechenden zu grunde zu legen. Man lese also:

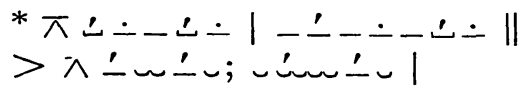

d.i. $\dot{-} \sim \dot{-} \sim$; dann als neuen verstypus schematisch ansetzen will.

d. i.

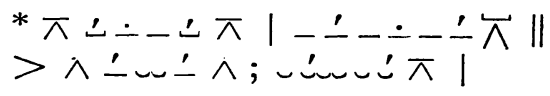

Ebenso unten:

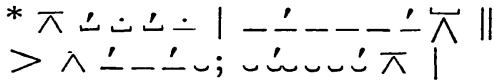

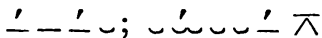

und auf s. 79 oben:

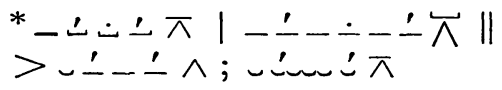

oder: $\because 1-1 \wedge ; \backsim \dot{\prime} \dot{1} \wedge$.

S. 80 sind in der analyse der pastourelle die pausensymbole der vordersätze umzustellen: $\pi \widehat{\wedge}$. Tripodien nimmt man besser nicht an.

Auf s. 82 ist vor dem schema des dekasyllabons die zweizeitige pause natürlich $\mathrm{zu}$ streichen.

S. 84. Zu den rhythmen der gruppe $A$ gehört, wie ich glaube, auch Walther, Lachm.39,11:

$$
\begin{aligned}
& \text { 1. } \pi \dot{1} \sim 1 \div-1 \div-\frac{1}{1} \cup, \cup 1-\frac{1}{1}-\frac{1}{1} \\
& \text { 2. }-\dot{1}-1-11 \\
& \text { 3. } \pi \dot{1}-1-1-1|-1-1| \pi \dot{1}-1-1-1 \\
& \text { Schema } \begin{array}{r}
a+b-c \\
a+b-c \\
d-w-d
\end{array}
\end{aligned}
$$

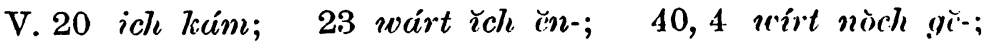

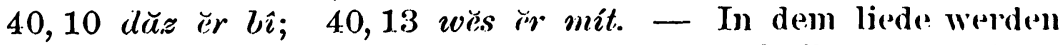
absichtlich die freiheiten einer ajteren technik nachgrahm. Vgl. auch Beitr. 23, 95. Der juhalt der mit hötischer minne nichts zu tun hat, stimmt genau dazu. 
S. 85. 7/ı B gehören lieder wie folgendes sehr künstliche von komrad von Wiirzburg, Bartsch 360, no. 9:

Meie den grïenen walt hat bekleit gar mit sîner giiete, daz ist wol schîn. Zweie sich jung und alt! âne leit ñz der boume blüete diu vogellîn Singent süezen sumersanc; dâ bî siht man wunne mê, Bluomen rôt, gel únde blanc dringent in touwe durh den grüenen klê. Der meie machet hôhen muot; dâ bî trûren swachet diu minne guot.

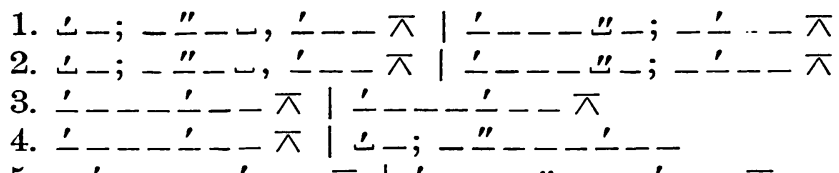

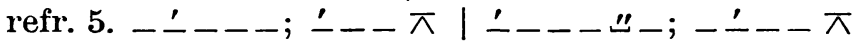

Ebenso Nîthart 14, 14:

Willekomen sî des meien schœne. ich hân vernomen, manegem senedem herzen trûren ist benomen.

Sorge lât, junge mägde, deist mîn rât. uns nâhet ein sumer; den enphâhet.

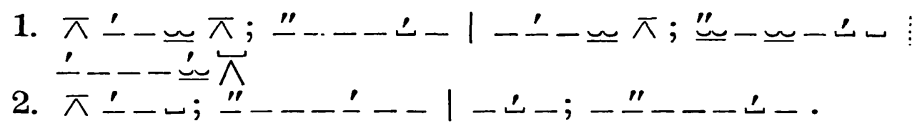

Man sieht wie der reim oft benutzt wird, die stellen der zusammenziehung anzudeuten.

S. 86. Zu MF. 127, 1. Wenn man in strophe 2, v. 1, 6 und str. 3, v. 6 vil streicht, stimmen die drei strophen hinsichtlich der auftaktverhältnisse genau überein. Str. 2 wäre dann zu lesen dér alsố und Nu ist. Vil konnte leicht zugesetzt werden.

S. 90 lies in str. 1 alscim mîn sélbes.

HALLE a. S., october 1898.

F. SARAN. 